\title{
FACTORING A GROUP AS AN AMALGAMATED FREE PRODUCT
}

\author{
EDWARD T. ORDMAN*
}

(Received 26 October 1971)

Communicated by G. E. Wall

Even if in a decomposition of a group

$$
G=\Pi^{*}\left(\left\{A_{i}, i \in I\right\} ; B\right)
$$

the $A_{i}$ are completely indecomposable, there may be another decomposition

$$
G=\Pi^{*}\left(\left\{C_{j}, j \in J\right\} ; D\right)
$$

with each $C_{j}$ properly contained in some $A_{i}$ and $D$ a proper subgroup of $B$. The example of Bryce ([1], p. 636) may be modified, at the cost of having one $A_{i}=B$, so that $I=J$ and $C_{i}<A_{i}$ for all $i$. It is our object to study this relationship between decompositions of a group.

In section 1 notation is introduced and an example of Stallings is expanded. In section 2 machinery motivated by the Van Kampen Theorem is constructed to show that the problems arising in section 1 may not be insurmountable. Section 3 contains an application of this machinery to extend a theorem of Holmes concerning lattices of subgroups.

\section{Decompositions of a group}

All results here apply to free products of arbitrarily many groups with a single amalgamated subgroup. To simplify notation, results are stated for a product with only two factors. The extension to more factors is immediate except for Theorems 5 and 6, which require slight rephrasing.

Let $G$ be a group and suppose $G$ is the free product of its subgroups $A$ and $B$ with the subgroup $C$ amalgamated. Then we write $G=(A * B ; C)$. The subgroup of $G$ generated by sets $R, S, T, \cdots$ and elements $a, b, c, \cdots$ will be denoted $(R, S, T, \cdots$, $a, b, c, \cdots)$. The group generated by elements $x, y, \cdots$ with relations $u=v, \cdots$, will be denoted $(x, y, \cdots \mid u=v, \cdots)$. Whether $(x, y)$ is the free group. or a subgroup of a group $G$, will be clear by the context.

* This work was supported by the National Science Foundation under Grant GP21186. 
Definition 1. Suppose $G=(A * B ; C)$ and $G=\left(A^{\prime} * B^{\prime} ; C^{\prime}\right)$, with $A \leqq A^{\prime}$, $B \leqq B^{\prime}, C \leqq C^{\prime}$. Then the first decomposition is called finer than the second, and the second coarser than the first. Among the immediate questions are: When can a decomposition be made finer? Are there minimal decompositions?

Lemma 1. Let $G=(A * B ; C)=\left(A^{\prime} * B^{\prime} ; C^{\prime}\right)$ with $A \leqq A^{\prime}, B \leqq B^{\prime}, C \leqq C^{\prime}$. If $A \neq A^{\prime}$ or $B \neq B^{\prime}$, then $C \neq C^{\prime}$, and conversely.

Proof. Since $C=A \cap B$ and $C^{\prime}=A^{\prime} \cap B^{\prime}$, it is clear that $C \neq C^{\prime}$ implies $A \neq A^{\prime}$ or $B \neq B^{\prime}$. In the forward direction, suppose

$$
A \cap B=C=C^{\prime}=A^{\prime} \cap B^{\prime},
$$

and suppose $x \in B^{\prime} \backslash B$. Now $x \notin A^{\prime}$ (otherwise $x \in C^{\prime}=C \subset B$ ) so $x \notin A$. Thus $x$ may be written as

$$
x=c g_{1} g_{2} . . g_{n}, n \geqq 2
$$

by the well known theorem of Schreier (cf. [3], p. 205), with $c \in C$ and $g_{i}$ alternately in $A \leqq A^{\prime}$ and $B \leqq B^{\prime}$, no $g_{i} \in C$. But in $G=\left(A^{\prime} * B^{\prime} ; C^{\prime}\right)$ this word is reducible to $x \in B^{\prime}$; hence some $g_{i} \in C^{\prime}$, contradicting $C^{\prime}=C$.

Corollary 1. Let $G=(A * B ;\{1\})$ be a free product. Then there is no strictly finer factorization of $G$.

Corollary 2. Let $G=(A * B ; C)$ and suppose $C$ is the center of $G$. Then there is no strictly finer factorization of $G$.

Proof. If $G=\left(A^{\prime} * B^{\prime} ; C^{\prime}\right)$ with $C^{\prime} \leqq C$, we have $C \leqq C^{\prime}$ since the amalgamated subgroup must contain the center.

Unfortunately, it is not true that $G=(A * B ;\{1\})$ is finer than any other decomposition of $G$, or even that any decomposition of a free product can be refined to be a free decomposition. This fact was pointed out by Stallings in [5] with the following example.

Example 1. (Stallings). Letting $(x, y)$ denote the free group on $x$ and $y$,

$$
(x, y)=\left(\left(x, y^{2} x^{2} y x^{-2} y^{-2}\right) *\left(x^{2}, y^{2}\right) ;\left(x^{2}, y^{2} x^{2} y^{2} x^{-2} y^{-2}\right)\right) .
$$

Further, no decomposition of $(x, y)$ finer than this one is free.

Stallings proves the second assertion by observing that if $(x, y)=(A * B ;\{1\})$ with $A \leqq\left(x, y^{2} x^{2} y x^{-2} y^{-2}\right)$ and $B \leqq\left(x^{2}, y^{2}\right), A \cap B=\{1\}$ and the subgroup generated by $A \cup B$ cannot contain $y$. He produces an isomorphism to show that the amalgamated free product in question is actually $(x, y)$; this may be done more routinely using Tietze transformations ([3], pp. 48ff). Denoting $x$, $y^{2} x^{2} y x^{-2} y^{-2}, x^{2}, y^{2}$ by $x, b, c, d$, the given decomposition may be written 


$$
\begin{aligned}
G & =\left((x, b) *(c, d) ;\left(x^{2}=c, b^{2}=d c d c^{-1} d^{-1}\right)\right) \\
& =\left(x, b, c, d \mid x^{2}=c, b^{2}=d c d c^{-1} d^{-1}\right) \\
& =\left(x, b, c, d, y \mid c=x^{2}, b^{2}=d x^{2} d x^{-2} d^{-1}, y=x^{-2} d^{-1} b d x^{2}\right) \\
& =\left(x, b, d, y \mid b^{2}=d x^{2} d x^{-2} d^{-1}, b=d x^{2} y x^{-2} d^{-1}\right) \\
& =\left(x, b, d, y \mid b=d x^{2} y x^{-2} d^{-1}, d x^{2} d x^{-2} d^{-1}=d x^{2} y^{2} x^{-2} d^{-1}\right) \\
& =\left(x, d, y \mid d=y^{2}\right) \\
& =(x, y) .
\end{aligned}
$$

This shows that $(x, y)$ has a decomposition which fails in an essential way to be free. Can such a decomposition of a free group be minimal? For example, can any refinement of Stallings' decomposition be minimal? I am unable to settle this question, but the following example seems to argue for the negative:

EXAMPLE 2. Let $A_{1}=\left(x, y^{2} x^{2} y x^{-2} y^{-2}\right), B=\left(x^{2}, y^{2}\right), \quad C_{1}=A_{1} \cap B$. If $A_{n}=\left(a_{n}, b_{n}\right)$, let $A_{n+1}=\left(b_{n}^{2} a_{n} b_{n}^{-2}, a_{n}^{2} b_{n} a_{n}^{-2}\right)$ and $C_{n+1}=A_{n+1} \cap B$. Then $(x, y)=\left(A_{n} * B ; C_{n}\right)$ for all $n$, and $A_{n+1}<A_{n}, C_{n+1}<C_{n}$ for all $n \geqq 1$.

The fact that $A_{n+1}<A_{n}$ is immediate, by consideration of word length. $C_{n+1} \leqq C_{n}$ follows since $C_{n}=A_{n} \cap B$, and $C_{n} \neq C_{n+1}$ will follow from Lemma 1 once we have shown $(x, y)=\left(A_{n} * B ; C_{n}\right)$ for all $n$. To do this, we apply an induction to the following statement:

Whenever a group $G$ is the free product of free subgroups $(a, b)$ and $(c, d)$ with amalgamated subgroup $(a, b) \cap(c, d)$ generated by $a^{2}=W(c, d)$ and $b^{2}=$ $V(c, d)$ (where $W$ and $V$ are words in $c$ and $d$ ), then $G$ is also the free product of its free subgroups $\left(b^{2} a b^{-2}, a^{2} b a^{-2}\right)$ and $(c, d)$ with amalgamated subgroup $\left(b^{2} a b^{-2}\right.$, $\left.a^{2} b a^{-2}\right) \cap(c, d)$ generated by $b^{2} a^{2} b^{-2}=V W V^{-1}$ and $a^{2} b^{2} a^{-2}=W V W^{-1}$.

That the intersection is correct may be proven by counting exponents. That the second amalgamated free product is in fact $G$ may be proven by using Tietze transformations:

$$
\begin{aligned}
G & =\left(r, s, c, d \mid r^{2}=V W V^{-1}, s^{2}=W V W^{-1}\right) \quad W=W(c, d), V=V(c, d) \\
& =\left(r, s, c, d, a, b \mid r^{2}=V W V^{-1}, s^{2}=W V W^{-1}, a=V^{-1} r V, b=W^{-1} s W\right) \\
& =\left(r, s, c, d, a, b \mid r=V a V^{-1}, s=W b W^{-1}, a^{2}=W, b^{2}=V\right) \\
& =\left(a, b, c, d \mid a^{2}=W, b^{2}=V\right) \\
& =\left((a, b) *(c, d) ;\left(a^{2}=W, b^{2}=V\right)\right) .
\end{aligned}
$$

This establishes the assertion of Example 2. It is worth noting that the intersection of all the $A_{n}$ is $\{1\}$, and that $B$ alone does not generate $(x, y)$. Thus, we have constructed a descending chain with no lower bound in the set of decompositions of $(x, y)$.

\section{General theorems}

The following theorem, motivated by the Van Kampen Theorem by way of [5], and proven in [4] (Theorem 3.2.2), gives a way of going from a finer to a coarser decomposition. 
THEOREM 1. Let $G=(A * B ; C)$. Let $S$ be a well ordered subset of $G$ such that if $s \in S$,

$$
s \in(A,\{r \in S \mid r<s\}) \cup(B,\{r \in S \mid r<s\}) .
$$

In particular, the first element of $S$ lies in $A \cup B$. Then $G=((A, S) *(B, S) ;(C, S))$.

The basic result of this section is a converse of Theorem 1; namely that any decomposition coarser than a given one may be obtained by adjoining such a set $S$ to each factor.

Lemma 2. Let $G=(A * B ; C)=\left(A^{\prime} * B^{\prime}, C^{\prime}\right), A \leqq A^{\prime}, B \leqq B^{\prime}, C<C^{\prime}$. Then either $A \cap\left(C^{\prime} \backslash C\right)$ or $B \cap\left(C^{\prime} \backslash C\right)$ is nonempty.

Proof. Let $h \in C^{\prime} \backslash C$, so that $h=c g_{1} \cdots g_{n}$ with the $g_{1}$ alternately in $A \backslash C$ and $B \backslash C$. The $g_{1}$ are alternately in $A^{\prime}$ and $B^{\prime}$, and $h \in C^{\prime}$ so some $g_{i} \in C^{\prime}$. Since this $g_{i}$ is in $A \backslash C$ or in $B \backslash C$, we are done.

Theorem 2. Let $G=\left(A^{*} B ; C\right)=\left(A^{\prime} * B^{\prime} ; C^{\prime}\right), A \leqq A^{\prime}, B \leqq B^{\prime}, C<C^{\prime}$. Then there is a well-ordered subset $S$ of $C^{\prime}$ such that if $s \in S$,

$$
s \in(A,\{r \in S \mid r<s\}) \cup(B,\{r \in S \mid<s\}),
$$

and such that $A^{\prime}$ is generated by $A \cup S, B^{\prime}$ by $B \cup S$ and $C^{\prime}$ by $C \cup S$.

Proof. Lemma 2 yields a first element $s_{1}$ for $S$. Using it, write

$$
G=\left(\left(A, s_{1}\right) *\left(B, s_{1}\right) ;\left(C, s_{1}\right)\right)
$$

by Theorem 1. Apply Lemma 2 to this decomposition to find $s_{2}$. Continue by transfinite induction to build up $S=\left\{s_{1}, s_{2}, \cdots\right\}$ (The limit steps are routine, since each intermediate $S$ satisfies the hypotheses for Theorem 1) until $(C, S)=C^{\prime}$, which happens after a number of steps not exceeding the cardinality of $C^{\prime} \backslash C$. Now by Theorem 1,

$$
G=((A, S) *(B, S) ;(C, S))
$$

Since $A \leqq A^{\prime}$ and $S \leqq C^{\prime} \leqq A^{\prime},(A, S) \leqq A^{\prime}$; similarly $(B, S) \leqq B^{\prime}$. Hence by Lemma $1,(A, S)=A^{\prime}$ and $(B, S)=B^{\prime}$.

As previously noted, the structure of the set of decompositions of $G$ is unpleasant; there are descending chains with no lower bounds, and pairs of decompositions with no common refinement. If we stay away from the bottom, however, we can find the following structure;

TheORem 3. Suppose $G=\left(G_{1} * G_{2} ; G_{0}\right)$, and consider the set of all decompositions of $G$ coarser than this one. Any two elements of this set have a greatest lower bound and a least upper bound in the set.

Proof. Suppose $G=\left(G_{1}^{1 *} G_{2}^{1} ; G_{0}^{1}\right)$ and $\left(G_{1}^{2} * G_{2}^{2} ; G_{0}^{2}\right)$ are the decompositions. We first construct a least upper bound. By Theorem 2, there is $S \subset G_{0}^{1}$ 
with $G_{i}^{1}=\left(G_{i}, S\right), i=0,1,2$. Now $G=\left(G_{1}^{2} * G_{2}^{2}: G_{0}^{2}\right)$ and $S$ satisfy the hypotheses of Theorem 1, so

$$
G=\left(\left(G_{1}^{2}, S\right) *\left(G_{2}^{2}, S\right) ;\left(G_{0}^{2}, S\right)\right)
$$

That this is the desired least upper bound is clear since

$$
\left(G_{i}^{2}, S\right)=\left(G_{i}^{2}, G_{i}, S\right)=\left(G_{i}^{2}, G_{i}^{1}\right)
$$

is the smallest subgroup of $G$ containing $G_{i}^{2} \cup G_{i}^{1}$.

We now construct a greatest lower bound. Denote $G_{0}^{1} \cap G_{0}^{2}$ by $B$. We now construct a set $S$ by transfinite induction. Let

$$
S_{1}=\left(G_{1} \cup G_{2}\right) \cap B
$$

and well-order it arbitrarily.

Let

$$
S_{\lambda+1}=\left(\left(G_{1}, S_{\lambda}\right) \cup\left(G_{2}, S_{\lambda}\right)\right) \cap B \text { for } \lambda \geqq 1 ;
$$

retain the order on $S_{\lambda} \subset S_{\lambda+1}$ and well-order the new elements arbitrarily to follow them. When this process terminates, denote the final $S_{\lambda}$ (which is the union of all all the $S_{\lambda}$ ) by $S_{0}$. Now

$$
G=\left(\left(G_{1}, S_{0}\right) *\left(G_{2}, S_{0}\right) ;\left(G_{0}, S_{0}\right)\right)
$$

is a lower bound for the original factorizations since $G_{i} \leqq G_{i}^{j}$ and $S_{0} \subset B \leqq G_{0}^{j}$ $\leqq G_{i}^{j}$. To show this is the greatest lower bound, suppose $G=\left(H_{1} * H_{2} ; H_{0}\right)$ is any other lower bound coarser than $G=\left(G_{1} * G_{2}, G_{0}\right)$. By Theorem $2, H_{0}=$ $\left(G_{0}, S\right)$ and

where

$$
S=S^{1} \cup S^{2} \cup \cdots \cup S^{\lambda} \cup \cdots
$$

$$
\begin{aligned}
& S^{1} \subset\left(G_{1} \cup G_{2}\right) \cap B=S_{1} \\
& S_{2} \subset\left(\left(G_{1}, S^{1}\right) \cup\left(G_{2}, S^{1}\right)\right) \cap B \subset\left(\left(G_{1}, S_{1}\right) \cup\left(G_{2}, S_{1}\right)\right) \cap B=S_{2}
\end{aligned}
$$

and by transfinite induction $S^{\lambda} \subset S_{\lambda}$, so $S \subset S_{0}$. Hence $H_{i}=\left(G_{i}, S\right) \subset\left(G_{i}, S^{0}\right)$ and our lower bound is coarser than any other.

\section{Products with normal amalgamation}

This section is due in large part to conversations with Professor Charles S. Holmes, in some of which Professor L. M. Sonneborn participated.

Let $G=(A * B ; C)$. Clearly $C$ is normal in $G$ if and only if it is normal in $A$ and in $B$. Now suppose $C$ is not normal in $G$. Then $C^{A}$ (the normal closure of $C$ in $A$ ) or $C^{B}$ is not $C$. Denote $C^{A} \cup C^{B}$ by $S_{1}$ and well-order it arbitrarily. By Theorem 1 ,

$$
G=\left(\left(A, S_{1}\right) *\left(B, S_{1}\right) ;\left(C, S_{1}\right)\right) .
$$

If $\left(C, S_{1}\right)$ is not normal in $G$, repeat the process; $S_{2}=\left(C, S_{1}\right)^{A^{\prime}} \cup\left(C, S_{1}\right)^{B^{\prime}}$, where $A^{\prime}=\left(A, S_{1}\right)$ and $B^{\prime}=\left(B, S_{1}\right) . S_{1} \subset S_{2}$, so we can well-order $S_{2}$ so that 
elements of $S_{2} \backslash S_{1}$ follow elements of $S_{1}$. Define $S_{\lambda}$ by transfinite induction, and let $S$ be the (suitably ordered) union of the $S_{2}$. Now

$$
G=((A, S *(B, S) ;(C, S))
$$

where $(C, S)$ is simply $C^{G}$, the normal closure of $C$ in $G$. Since $(A, S)=(A, C, S)=$ $\left(A, C^{G}\right)$, we have proven:

TheOREM 4. Let $G=(A * B ; C)$. Then

$$
G=\left(\left(A, C^{G}\right) *\left(B, C^{G}\right) ; C^{G}\right) .
$$

Since $C^{G}$ is normal in $\left(A, C^{G}\right)$, we may compute its index.

Lemma 3. $\left[\left(A, C^{G}\right): C^{G}\right]=\left[A ; C^{A}\right]$, and similarly for $B$.

Proof. This may be proven by using the word problem, or by first looking at the isomorphism

$$
\frac{\left(A^{*} B ; C\right)}{C^{G}} \cong \frac{A}{C^{A}} * \frac{B}{C^{B}}
$$

from which it is clear that $C^{A}=A \cap C^{G}$. Then since $C^{G}$ is normal in $G$,

$$
\frac{A}{C^{A}}=\frac{A}{A \cap C^{G}} \cong \frac{\left(A, C^{G}\right)}{C^{G}} \text {. }
$$

We are now able to extend somewhat the following theorem of Holmes [2].

Theorem 5. Let $G=\left(A^{*} B ; C\right), C$ normal in $G, A \neq C \neq B,[A: C]>2$ or $[B: C]>2$. Then $G$ is determined by its lattice of subgroups.

Our extension is:

TheOREM 6. Let $G=\left(A^{*} B ; C\right),\left[A ; C^{A}\right]>2,\left[B ; C^{B}\right] \geqq 2$. Then $G$ is determined by its lattice of subgroups.

\section{References}

[1] R. A. Bryce, 'A note on free products with normal amalgamation,' J. Austral. Math. Soc. 8 (1968), 631-637.

[2] Charles S. Holmes, 'Projectivities of free products,' Rend. Sem. Math. Univ. Padova. 42 (1969), 341-387.

[3] W. Magnus, A. Karrass, D. Solitar, Combinatorial Group Theory (Interscience, New York, 1966).

[4] E. T. Ordman, 'On subgroups of amalgamated free products,' Proc. Cambridge Philos. Soc. 69 (1971), 13-23.

[5] J. R. Stallings, 'A topological proof of Grushko's theorem on free products,' Math. Z. 90 (1965), $1-8$.

University of Kentucky

Lexington, Kentucky, 40506

U.S.A. 